\title{
The Footprints of Gut Microbial-Mammalian Co-Metabolism
}

\author{
Xiaojiao Zheng, ${ }^{+,}$Guoxiang Xie, ${ }^{\ddagger}$ Aihua Zhao, ${ }^{\dagger}$ Linjing Zhao, ${ }^{\dagger}$ Chun Yao, ${ }^{\ddagger}$ Norman H. L. Chiu, ${ }^{\S}$ \\ Zhanxiang Zhou, ${ }^{\ddagger}$ Yuqian Bao," Weiping Jia, ${ }^{*, l l}$ Jeremy K. Nicholson, ${ }^{\perp}$ and Wei Jia, ${ }^{*,}$ \\ ${ }^{\dagger}$ School of Pharmacy, Shanghai Jiao Tong University, Shanghai 200240, P. R. China \\ ${ }^{\ddagger}$ Department of Nutrition, University of North Carolina at Greensboro, North Carolina Research Campus, Kannapolis, \\ North Carolina 28081, United States \\ ${ }^{\S}$ Department of Chemistry and Biochemistry, University of North Carolina at Greensboro, Greensboro, North Carolina 27402, \\ United States \\ "Department of Endocrinology and Metabolism, Shanghai Jiao Tong University Affiliated Sixth People's Hospital, Shanghai Diabetes \\ Institute, Shanghai Clinical Center of Diabetes, Shanghai 200233, P. R. China \\ ${ }^{\perp}$ Biomolecular Medicine, Department of Surgery and Cancer, Faculty of Medicine, Imperial College, London SW7 2AZ, \\ United Kingdom \\ S Supporting Information
}

ABSTRACT: Gut microbiota are associated with essential various biological functions in humans through a "network" of microbial-host co-metabolism to process nutrients and drugs and modulate the activities of multiple pathways in organ systems that are linked to different diseases. The microbiome impacts strongly on the metabolic phenotypes of the host, and hence, metabolic readouts can give insights into functional metagenomic activity. We applied an untargeted mass spectrometry (MS) based metabonomics approach to profile normal Wistar rats exposed to a broad spectrum $\beta$-lactam antibiotic imipenem/cilastatin sodium, at $50 \mathrm{mg} / \mathrm{kg} /$ daily for 4 days followed by a 14-day recovery period. In-depth metabolic phenotyping allowed identification of a panel of 202 urinary and 223 fecal metabolites significantly related to end points of a functional metagenome ( $p<0.05$ in at least one day), many of which have not been previously reported such as oligopeptides and carbohydrates. This

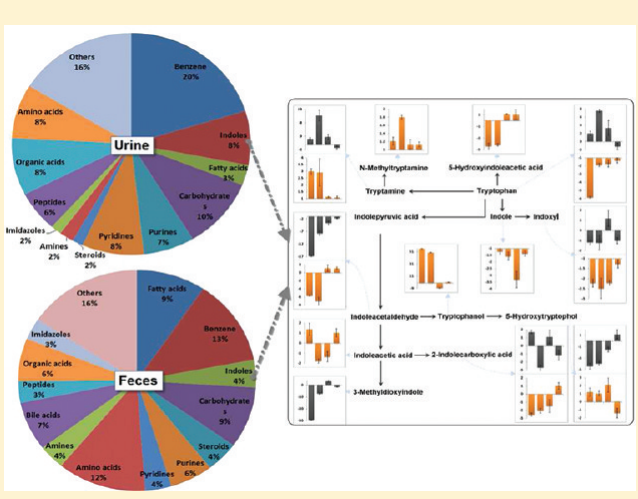
study shows extensive gut microbiota modulation of host systemic metabolism involving short-chain fatty acids, tryptophan, tyrosine metabolism, and possibly a compensatory mechanism of indole-melatonin production. Given the integral nature of the mammalian genome and metagenome, this panel of metabolites will provide a new platform for potential therapeutic markers and mechanistic solutions to complex problems commonly encountered in pathology, toxicology, or drug metabolism studies.

KEYWORDS: gut microbial-mammalian co-metabolism, microbiome, metabonomics, metabolomics, metagenomics, antibiotics, mass spectrometry

\section{INTRODUCTION}

Human symbiotic gut microorganisms perform a variety of useful functions via interactive microbial-host co-metabolism and signaling, which are closely involved with digestion of unutilized energy substrates to generate biologically important metabolites such as short-chain fatty acids (SCFAs), biotin and vitamin $\mathrm{K}$, stimulate cell growth, and also repress the growth of harmful microorganisms, thereby defending against some diseases. ${ }^{1-3}$ We have recently proposed a therapeutic strategy of manipulating the gut microbiome so that the dysregulation of the microbial-mammalian co-metabolism associated with a pathological state can be reversed. ${ }^{4}$ By using this strategy, the gut microbiotic environment could therefore be a new source of drug targets for the treatment of metabolic disorders, various conditions in the gastrointestinal (GI) tract and also neurological disorders. ${ }^{5}$

The variation in the GI metabolism is related directly to the activities of various microorganisms that coexist in the gut. With the recent advances in metagenomics ${ }^{6}$ based on $16 \mathrm{~S}$ rRNA gene sequence methods, ${ }^{7-9}$ the direct correlation of global metabolic footprints with gut microbiome becomes technically feasible with an aim to decipher the host-microbe relationships and to discover novel molecular targets in the microbial-mammalian metabolic axis. Our recent study of human gut microbiota using metabolic profiling coupled with the metagenomic sequencing provided a basis for understanding the "microbial-mammalian metabolic axis", which led to the concept of "functional metagenomics", defined as "the characterization of key functional members of the microbiome that most influence host metabolism and hence health". ${ }^{10}$

Given the integral nature of a specific gut microbiome and metabolome, any differences in the microbial communities could

Received: August 17, 2011

Published: October 04, 2011 
result in significant alterations in the extracellular metabolome (metabolic footprints), which may account for important findings commonly encountered in pathology, toxicology or drug metabolism studies. Metabonomics (or metabolomics), an array of analytical techniques including high-resolution nuclear magnetic resonance (NMR) spectroscopy ${ }^{11}$ and chromatographymass spectrometry (MS), ${ }^{12,13}$ enables comprehensive characterization of the metabolic footprints of mammalian hosts that can be correlated to the microbial community in the intestinal tract. $^{14,15}$ Recent studies by Nicholson et al. ${ }^{16-18}$ demonstrate that the metabolic variations in GI compartments such as duodenum, jejunum, and ileum, mammalian tissues from kidney and liver, and biofluids such as blood and urine are directly related to the activities of various microorganisms that coexist in the gut.

Here, we report a profiling study of urinary and fecal metabolites in Wistar rats exposed to a broad spectrum $\beta$-lactam antibiotic, Imipenem/cilastatin sodium, in a specific pathogenfree (SPF) environment, using both gas chromatography-mass spectrometry (GC-MS) and liquid chromatography-mass spectrumetry (LC-MS). Urine and feces samples were collected at day 0 (predose), days $1-4$ during antibiotic treatment, and days $5,8,12$, and 18 of recovery period. The aim of this study is to provide important baseline information of a complete spectrum of metabolites produced from the microbial-mammalian co-metabolism, to improve our understanding of the "functional metagenome" as well as molecular mechanisms underlying multilevel host - microbe interactions.

\section{MATERIALS AND METHODS}

\section{Chemicals}

Imipenem/cilastatin sodium (with equal quantities, trade named Tienam), a broad spectrum $\beta$-lactam antibiotic, was purchased from China National Pharmaceutical Group Corporation (Shanghai, China) and authorized for experimental use by School of Pharmacy, Shanghai Jiao Tong University (Shanghai, China). It was prepared in ultrapure water (Milli-Q system, Millipore, Billerica, MA) at a concentration of $5 \mathrm{mg} / \mathrm{mL}$. Methanol, acetonitrile, formic acid, chloroform and pyridine with HPLC grade were purchased from Merck Chemicals (Darmstadt, Germany). p-Chlorophenylalanine, urease (30 U, Type C), BSTFA (1\% TMCS), methoxyamine, leucine-enkephalin and all of the mammalian standards used for compound annotation and mix standards were obtained from Sigma-Aldrich (St. Louis, MO).

\section{Animal Handling and Sample Collection}

The animal study was performed at the Center for Laboratory Animals, Shanghai Jiao Tong University, Shanghai, China. A total of 12 male Wistar rats (4 weeks old; $78-88 \mathrm{~g}$ ) were purchased from Shanghai Laboratory Animal Co, Ltd. (SLAC, Shanghai, China). All rats were housed in a specific-pathogen-free (SPF) environment, under a controlled condition of $12 \mathrm{~h}$ light/ $12 \mathrm{~h}$ dark cycle at $20-22{ }^{\circ} \mathrm{C}$ and $45 \pm 5 \%$ humidity, with free access to chow and water. After one-week accommodation in metabolic cages, the rats were randomly divided into 2 groups: antibioticperturbed group $(n=6)$, dosed with antibiotic Imipenem/ cilastatin sodium at a daily dose of $50 \mathrm{mg} / \mathrm{kg}$ of body weight from day 1 to day 4 and control group $(n=6)$, which received the same volume of water, antibiotic vehicle, from day 1 to day 4 . After antibiotic perturbation, all rats were housed in a controlled environment for another 14 days as a recovery period. The volume of water and chow consumed and body weight of each rat were recorded carefully every day. Daily 18-h (20:00 p.m.-14:00 p.m. on the next day) urine and feces (19:00 p.m.) samples were collected throughout the experiment and frozen $\left(-80{ }^{\circ} \mathrm{C}\right)$ stored until analysis. Samples were collected at day 0 (predose), days $1-4$ during antibiotic treatment, and days 5, 8, 12 , and 18 of recovery period for metabonomic analysis.

\section{Sample Preparation}

Urine samples were prepared using the protocol established by our lab, ${ }^{19,20}$ and feces samples were extracted according to that described in published papers ${ }^{21}$ with minor modification.

\section{Metabonomic Profiling with LC-MS and GC-MS}

A Waters ACQUITY ultra performance liquid chromatography (UPLC) system equipped with a binary solvent delivery manager and a sample manager (Waters Corporation, Milford, $\mathrm{MA}$ ), coupled to a tandem quadrupole-time-of-flight (Q-TOF) mass spectrometry equipped with an electrospray interface (Waters Corporation, Milford, MA) was used as a LC-MS platform. An Agilent 6890N gas chromatography coupled with a Pegasus HT time-of-flight (TOF) mass spectrometry (Leco Corporation, St. Joseph, MI) was used as the GC-MS platform. The samples were run in the order of "control-antibioticcontrol”, alternately, to minimize systematic analytical deviations. Mixed standards sample as quality control was injected every 10 injections.

\section{Spectral Data Analysis}

The MS data of urine and feces samples collected at different time points were analyzed to identify potential discriminate variables. For LC-MS, the raw data were analyzed by the MarkerLynx applications manager version 4.1 (Waters, Manchester, U.K.). The parameters used were set as follows: initial retention time $=0.00 \mathrm{~min}$, final retention time $=9.5 \mathrm{~min}$ (as the rest time was for column equilibration), mass tolerance $(\mathrm{Da})=0.02$, peak width at $5 \%$ height was auto, peak to peak baseline noise was auto, intensity threshold $=50$, mass window $=0.02$, retention time window $=0.2 \mathrm{~min}$, and noise elimination level $=6$. The resulting matrix contained arbitrarily assigned peak index (retention time $(\mathrm{Rt})-m / z$ pairs), sample names and peak intensity. After data processing, a list of the intensities of the peaks detected was generated for the first sample, using Rt and $\mathrm{m} / \mathrm{z}$ data pairs as the identifier of each peak. The acquired MS files from GC-MS analysis were exported in NetCDF format by ChromaTOF software (v3.30, Leco Co., Los Angeles, CA). CDF files were extracted using custom scripts (revised Matlab toolbox HDA, developed by Par Jonsson, et al. ${ }^{22}$ ) in the MATLAB 7.1 (The MathWorks, Inc., Natick, MA) for data pretreatment procedures such as baseline correction, denoising, smoothing, and alignment; time-window splitting; and peak feature extraction (based on multivariate curve resolution algorithm). The resulting three dimension data set included sample information, peak retention time and peak intensities.

\section{Compound Identification}

Compound identification was performed using our in-house library containing $\sim 800$ mammalian metabolite standards. For LC-MS generated data, identification was performed by comparing with the accurate mass and Rt of reference standards in our in-house library and the accurate mass of compounds obtained from the web-based resources such as the Human Metabolome Database (http://www.hmdb.ca/) and METLIN (http://metlin.scripps.edu). For GC-MS generated data, 

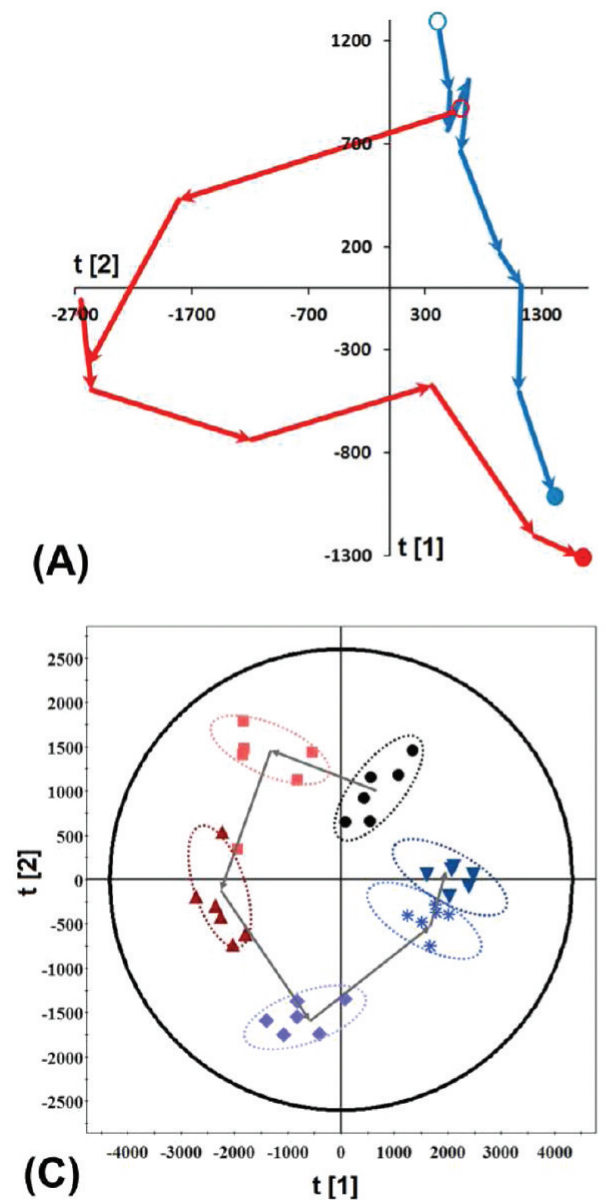

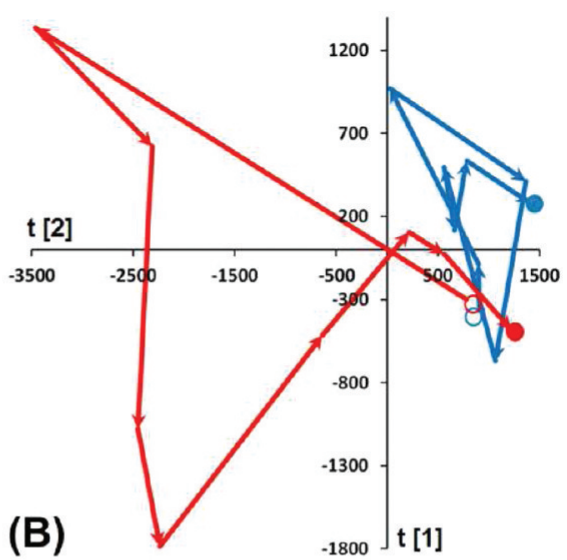

$\rightarrow$ Control
$\rightarrow$ Antibiotic

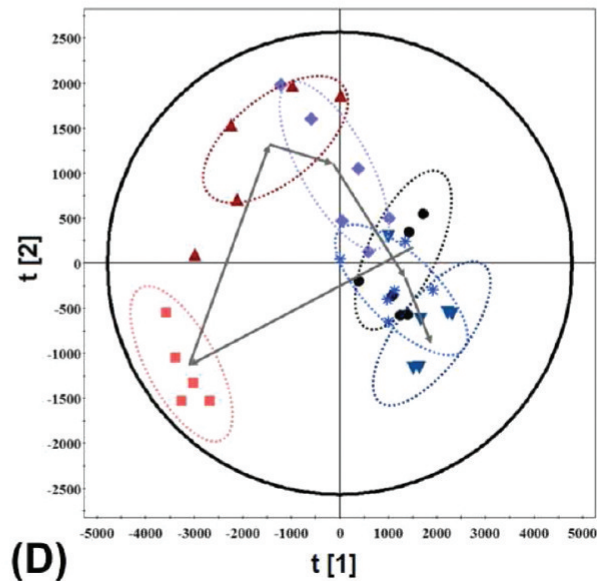

- Day 0

Day 1

$\triangle$ Day 3

Day 5

* Day 12

$\checkmark$ Day 18

Figure 1. Alteration of metabolome shown by trajectories and PLS-DA plots. Scores plots of PLS-DA are derived from (A) urinary and (B) fecal metabonomic (combined GC-MS and LC-MS) data for the control group (blue) and the antibiotic group (red) across the time course of predose (day 0), antibiotic treatment (days 1-4) and recovery period (days 5-18). Each dot in the plots represents mean value of the scores from the first and second principal components at a time point. The circle and dot denote the starting point (predose, day 0) and the end point (day 18), respectively. PLS-DA scores plots of (C) urinary and (D) fecal metabolic profiles show similar time-dependent changes of the antibiotic group at different time points.

identification was processed by comparing with the mass fragments and Rt of reference standards in our in-house library or mass fragments with NIST 05 Standard mass spectral databases in NIST MS search 2.0 (NIST, Gaithersburg, MD) software with a similarity of more than $70 \%$.

\section{Statistical Analysis}

Internal standards and any known artificial peaks, such as peaks caused by noise, column bleed and BSTFA derivatization procedure, were removed from the data sets obtained in above Section (Spectral Data Analysis). Urine data were normalized to the total intensity, while feces data did not need to be normalized since the extraction solvent adding in feces depended on the weight of feces, keeping the same feces concentration of different weights of samples. Data were then mean centered and unit variance scaled in a columnwise manner in the Simca-P 12.0 Software package (Umetrics, Umeå, Sweden). PLS-DA was carried out to get the three-dimensional discriminations and trajectories by score parameters after combining the data from two different instruments of the same sample. All the metabolites identified were processed at a univariate analysis using a two-tailed unpaired $t$ test with the critical $p$ value set to 0.05 . The fold changes were the intensity mean value ratio of antibiotic group to control group in the corresponding day. Heat-maps were created to visualize the metabolites derived from gut microbial-mammalian co-metabolism and $t$ test was carried out in MATLAB 7.1 (The MathWorks, Inc., Natick, MA). Additionally, KEGG Global Pathway Map was constructed in Cytoscape 2.8.0. ${ }^{23}$

\section{RESULTS}

Effect of Antibiotic Intervention in Urine and Feces

Experimental rats displayed diarrhea throughout the 4 day antibiotic treatment period. The diarrhea incidence was reduced after the first day of antibiotic withdrawal and stopped 6 days before the end of experiment. The metabolic alteration caused by the microbial alterations after antibiotic treatment was substantial. The complex metabolic effects and dynamic changes of microbial manipulation were visualized by comparing the antibiotic treated rats to nonantibiotic control rats. The timedependent metabolic "footprints" in urine and feces of experimental rats resulting from antibiotic exposure are illustrated in Figure 1 across the 4-day treatment period and the 14-day recovery period with the criterion described by Holmes et al. ${ }^{24}$ The effect of Imipenem on the gut microbiota was profound and 


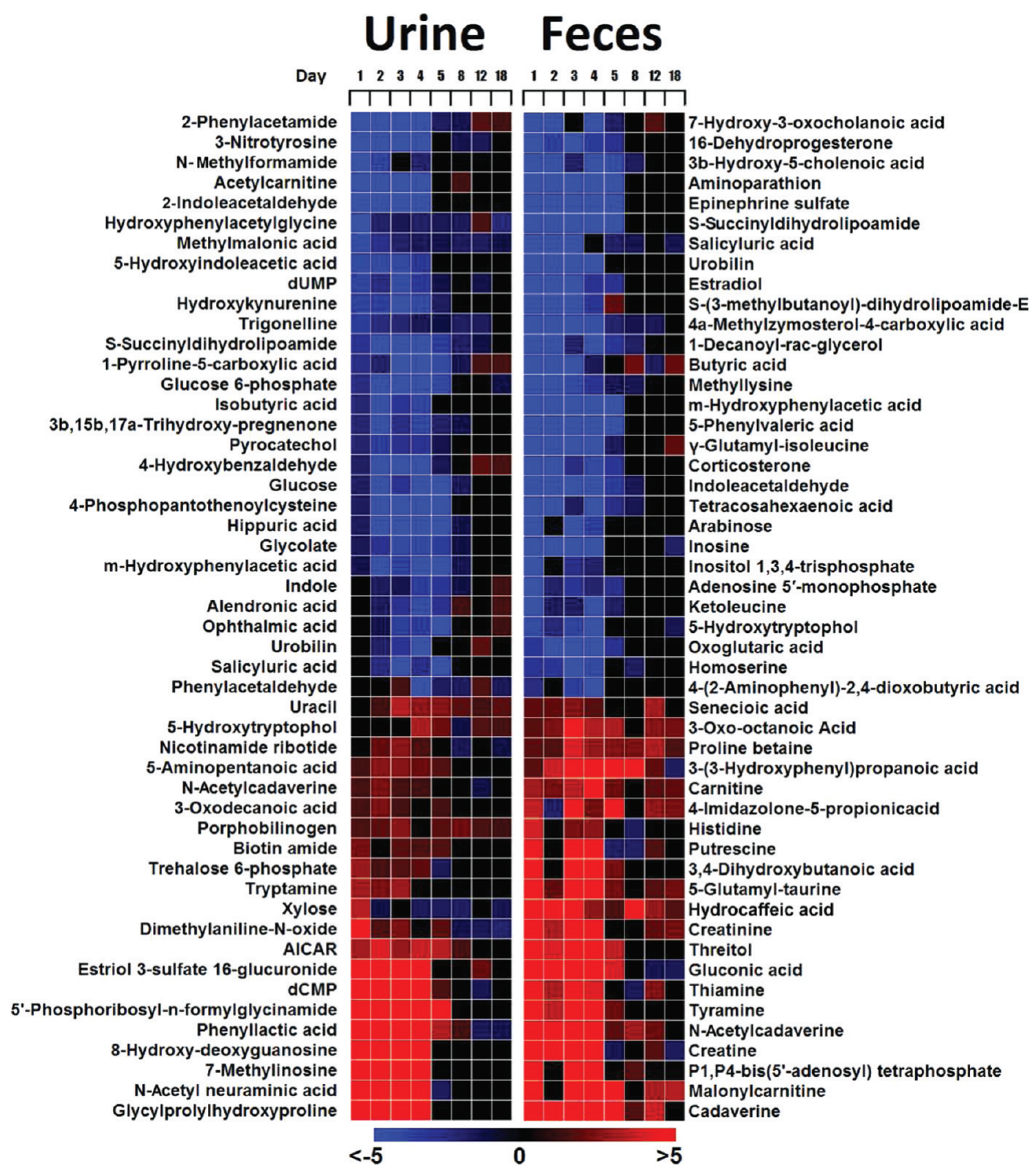

Figure 2. Heat-maps of representative urinary and fecal metabolites involved in gut microbial-mammalian co-metabolism. Heat-maps show the timedependent fluctuations (red $=$ increased concentration, blue $=$ decreased concentration) of (left) urinary and (right) fecal metabolites as a result of microbial perturbation. Each cell in the heat-maps represents the fold change of a particular metabolite, which is the ratio of the concentration in the antibiotic group to that in the control group.

rapid, with most significant changes in metabolic profile from the day prior to the antibiotic treatment to day 1 . The trajectories show that the urinary and fecal metabolic changes (Figure 1) underwent a significant shift from day 0 to day 1 , continued to "drift away" from day 1 to day 5, and gradually recovered with a steady pattern approaching the control (nonantibiotic) group. The metabolic trajectories generated from LC-MS or GC-MS based metabonomic data (Supplementary Figure 1, Supporting Information) are similar to that of combined data set shown in Figure 1. PLS-DA scores plots $\left((C) \mathrm{R}^{2} \mathrm{X}=0.36, \mathrm{R}^{2} \mathrm{Y}=0.545\right.$, $\mathrm{Q}^{2} \mathrm{Y}=0.37$; (D) $\mathrm{R}^{2} \mathrm{X}=0.744, \mathrm{R}^{2} \mathrm{Y}=0.969, \mathrm{Q}^{2} \mathrm{Y}=0.616$ ) showed the dynamic change in the antibiotic group within the selected days. A clear differentiation of the antibiotic perturbation can be observed that the points of each animal in the first, third and fifth day clustered, respectively, far from the predose points. Those points representing day 12 and day 18 were close to the predose points, showing a normalization of metabolic profiles after day 12. The trajectories of both urinary and fecal metabolic profiles in Figure 1 suggest that the experimental rats in the antibiotic treatment group were able to restore their microbial community composition after a 2 -week recovery period. This is also evidenced by the heat-maps of the urinary and fecal metabolites showing metabolic fluctuations normalized from day $8-18$ in Figure 2 .

\section{Altered Metabolite Patterns in Urine and Feces}

To characterize the metabolic perturbation in response to antibiotic intervention in urine and feces, representative 
Table 1. Representative Urinary and Fecal Metabolites Derived from Gut Microbial-Mammalian Co-metabolism ${ }^{a}$

\begin{tabular}{|c|c|c|}
\hline metabolic pathway & urinary metabolites & fecal metabolites \\
\hline Tryptophan Metabolism & $\begin{array}{l}\text { Tryptophan } \\
\text { 5-Hydroxytryptophol } \\
\text { Tryptamine } \\
\text { 2-Indoleacetaldehyde } \\
\text { 2-Indolecarboxylic acid } \\
\text { LC3 } \\
\text { 6-Hydroxymelatonin sulfate } \\
\text { Tryptophanol }^{\mathrm{LC} 3} \\
\text { Indoleacetic acid }^{\mathrm{LC} 1} \\
\text { Indolelactic acid }^{\mathrm{LC} 3} \\
\text { Indole }^{\mathrm{LC} 3} \\
\text { N-Methyltryptamine } \\
\text { Kynurenic acid }^{\mathrm{LC} 3} \\
\text { Indoxyl sulfate }^{\mathrm{GC} 1} \\
\text { Hydroxykynurenine }^{\mathrm{LC} 1} \\
\text { Nicotinamide }^{\mathrm{GC} 1}\end{array}$ & $\begin{array}{l}\text { Tryptophan } \\
\text { 5-Hydroxytryptophol } \\
\text { Tryptamine }^{\mathrm{LC} 3} \\
\text { 2-Indoleacetaldehyde } \\
\text { 2-Indolecarboxylic acid } \\
\text { 6-Hydroxymelatonin sulfate } \\
\text { Nicotinic acid }\end{array}$ \\
\hline Tyrosine and Phenylalanine Metabolism & $\begin{array}{l}\text { Tyrosine }^{\mathrm{LC} 3} \\
\text { 2-Phenylacetamide } \\
\text { Phenylethanolamine }^{\mathrm{LC} 3} \\
\text { Phenylacetaldehyde }^{\mathrm{LC} 3} \\
\text { Phenylacetic acid }^{\mathrm{LC} 3} \\
\text {-Hydroxyphenylacetic acid }^{\mathrm{GC} 1} \\
\text { m-Hydroxyphenylacetic acid } \\
\text { p-Hydrocinnamic acid } \\
\text { Shikimic acid }^{\mathrm{GC} 1} \\
\text { Phenol } \\
\text { Thyronine }^{\mathrm{LC} 1} \\
\text { p-Hydroxybenzoic acid } \\
\text { Hippuric acid }^{\mathrm{GC} 1} \\
\text { Melanin }^{\mathrm{LC} 3} \\
\text { Dopamine }^{\mathrm{LC} 1} \\
\text { Homovanillic acid }^{\mathrm{GC} 1} \\
\text { Normetanephrine }^{\mathrm{LC} 1}\end{array}$ & $\begin{array}{l}\text { Tyrosine } \\
\text { 2-Phenylacetamide } \\
\text { Phenylethanolamine }^{\mathrm{LC} 3} \\
\text { Phenylacetaldehyde }^{\mathrm{LC} 3} \\
\text { Phenylacetic acid }^{\mathrm{LC} 3} \\
\text { p-Hydroxyphenylacetic acid }^{\mathrm{GC} 1} \\
\text { m-Hydroxyphenylacetic acid } \\
\text { p-Hydrocinnamic acid }^{\mathrm{GC} 2} \\
\text { Phenylalanine }^{\mathrm{GC} 1} \\
\text { Tyramine }^{\mathrm{GC} 1} \\
\text { Dopa }^{\mathrm{LC} 1} \\
\text { Hydrocaffeic acid }^{\mathrm{GC} 2} \\
\text { Hydroxydopamine }^{\mathrm{LC} 1} \\
\text { Epinephrine sulfate }^{\mathrm{LC} 3}\end{array}$ \\
\hline Organic Acids Metabolism & $\begin{array}{l}\text { Butyric acid } \\
\text { Isobutyric acid }^{\mathrm{LC} 3} \\
\text { Lactic acid }^{\mathrm{GC} 1} \\
\text { 2-Methylbutyric acid } \\
\text { Hydroxyisobutyric acid }^{\mathrm{GC} 1} \\
\text { Propionic acid }^{\mathrm{GC} 3}\end{array}$ & $\begin{array}{l}\text { Butyric acid } \\
\text { Isobutyric acid }^{\mathrm{LC} 2} \\
\text { Lactic acid }^{\mathrm{GC} 1} \\
\text { 3-Hydroxybutyric acid } \\
\text { 4-Aminobutyric acid } \\
\text { Caprylic acid } \\
\text { Myristic acid }^{\mathrm{GC} 1} \\
\text { Palmitic acid }^{\mathrm{GC} 1} \\
\text { Suberic acid }\end{array}$ \\
\hline Oligopeptides & $\begin{array}{l}\text { Aspartyl-glutamic acid }{ }^{\mathrm{LC} 3} \\
\text { Aspartyl-leucine } \\
\text { Aspartyl-serine } \\
\text { Leucyl-proline }^{\mathrm{LC} 3} \\
\text { Phenylalanyl-hydroxyproline } \\
\text { Prolyl-glycine }^{\mathrm{LC} 3}\end{array}$ & \\
\hline Carbohydrate Metabolism & $\begin{array}{l}N \text {-acetyl-glucosamine }{ }^{\mathrm{GC} 2} \\
\text { Glucose }^{\mathrm{GC} 2} \\
\text { Glucose 6-phosphate }^{\mathrm{LC} 1} \\
\text { Myo-inositol } \\
\text { Threonic acid } \\
\text { Arabitol }^{\mathrm{LC} 1} \\
\text { Arabinose }^{\mathrm{LC} 1} \\
\text { Xylitol }^{\mathrm{GC} 1} \\
\text { Xylose }^{\mathrm{GC} 1} \\
\text { Ribose }^{\mathrm{GC} 1} \\
\text { Fructose }^{\mathrm{GC} 1}\end{array}$ & $\begin{array}{l}N \text {-acetyl-glucosamine } \\
\text { Glucose }^{\mathrm{GC} 1} \\
\text { Glucose }^{\mathrm{LC}} \text {-phosphate } \\
\text { Myo-inositol } \\
\text { Threonic acid } \\
\text { The3 } \\
\text { Arabitol }^{\mathrm{GC} 1} \\
\text { Arabinose }^{\mathrm{LC} 1} \\
\text { Xylitol }^{\mathrm{GC} 1} \\
\text { Xylose }^{\mathrm{LC} 1} \\
\text { Ribose }^{\mathrm{GC1}} \\
\text { Fucose }^{\mathrm{GC} 1} \\
\text { Rhamnose }^{\mathrm{GC} 1} \\
\text { Gluconic acid }^{\mathrm{GC} 2}\end{array}$ \\
\hline
\end{tabular}


Table 1. Continued

Purine and Pyrimidine Metabolism

Histidine Metabolism

Cysteine and Methionine Metabolism

Valine, Leucine and Isoleucine Metabolism

Lysine Metabolism

Arginine and Proline Metabolism

\author{
Hypoxanthine ${ }^{\mathrm{LC} 1}$ \\ Allantoin $^{\mathrm{GC} 1}$ \\ Methylguanine $\mathrm{LC1}^{\mathrm{LC}}$ \\ Thymine ${ }^{\text {LC1 }}$ \\ Deoxycytidine ${ }^{\mathrm{LC} 1}$ \\ Cytosine GC1 $^{\text {GC1 }}$ \\ Uracil $^{\mathrm{GC} 1}$ \\ $\beta$-Alanine ${ }^{\mathrm{GC} 1}$ \\ Purine ${ }^{\mathrm{LC} 1}$ \\ 5-Aminoimidazole ${ }^{\text {LC3 }}$ \\ Adenosine $\mathrm{e}^{\mathrm{LC} 1}$ \\ Guanosine $\mathrm{L}^{\mathrm{LC} 1}$ \\ Methylmalonic acid ${ }^{\mathrm{GC1}}$ \\ Deoxyuridine ${ }^{\mathrm{LC1}}$ \\ Malonic acid ${ }^{\mathrm{GC} 1}$ \\ Orotic acid ${ }^{\mathrm{GC} 1}$ \\ Urocanic acid ${ }^{\mathrm{LC} 3}$ \\ L-Methylhistidine $\mathrm{LC1}^{\mathrm{LC}}$
}

Cysteine $\mathrm{GC1}^{\mathrm{GC}}$

2-Oxo-4-methylthiobutyric acid ${ }^{\mathrm{LC} 3}$

Hypotaurine GC1

5-Glutamyltaurine $\mathrm{LC3}^{\mathrm{LC}}$

O-Phosphoserine ${ }^{\mathrm{LC} 1}$

Pyroglutamic acid ${ }^{\mathrm{LC} 1}$

Homocysteine ${ }^{\text {LC1 }}$

Methionine ${ }^{\text {LC1 }}$

Urea $^{\mathrm{GC} 1}$

Isoleucine $\mathrm{GC1}^{\mathrm{GC}}$

Leucine ${ }^{\mathrm{GC} 1}$

Lysine ${ }^{\mathrm{LC} 1}$

Glycine $e^{\mathrm{LC} 1}$

Glutaric acid $^{\mathrm{GC} 1}$

Cadaverine ${ }^{\mathrm{GC} 1}$

5-Aminopentanoic acid ${ }^{\mathrm{GC} 2}$

Glutamine ${ }^{\mathrm{GC} 1}$

Ornithine $\mathrm{GC1}^{\mathrm{GC}}$

Creatinine ${ }^{\mathrm{GC} 1}$

Proline ${ }^{\mathrm{LC} 1}$
Hypoxanthine LC1 $^{\text {LC }}$

Allantoin ${ }^{\mathrm{GC} 2}$

Methylguanine ${ }^{\text {LC1 }}$

Thymine ${ }^{\text {LC1 }}$

Deoxycytidine $^{\mathrm{GC} 2}$

Cytosine $\mathrm{LC}^{\mathrm{LC}}$

Uracil $^{\mathrm{LC1}}$

$\beta$-Alanine ${ }^{\mathrm{GC} 1}$

Phosphoribosylformylglycineamidine ${ }^{\text {LC3 }}$

Inosine ${ }^{\mathrm{LC} 3}$

$\mathrm{dIDP}^{\mathrm{LC} 3}$

Xanthine ${ }^{\mathrm{LC} 1}$

Xanthosine $\mathrm{e}^{\mathrm{LC} 3}$

Adenosine $5^{\prime}$-monophosphate ${ }^{\text {LC1 }}$

Adenine $^{\mathrm{LC} 1}$

Cytidine $^{\mathrm{LC} 1}$

Carbamoyl aspartate ${ }^{\mathrm{LC} 3}$

Urocanic acid ${ }^{\mathrm{LC} 3}$

Histidine ${ }^{\text {LC1 }}$

4-Imidazolone-5-propionic acid ${ }^{\mathrm{LC} 3}$

Imidazole-4-acetaldehyde ${ }^{\mathrm{LC} 3}$

Imidazoleacetic acid ${ }^{\mathrm{LC} 3}$

Methylimidazoleacetic acid ${ }^{\mathrm{LC} 3}$

Cysteine ${ }^{\text {GC1 }}$

2-Oxo-4-methylthiobutyric acid ${ }^{\mathrm{LC} 3}$

Hypotaurine ${ }^{\mathrm{LC} 1}$

5-Glutamyltaurine $\mathrm{LC}^{\mathrm{LC}}$

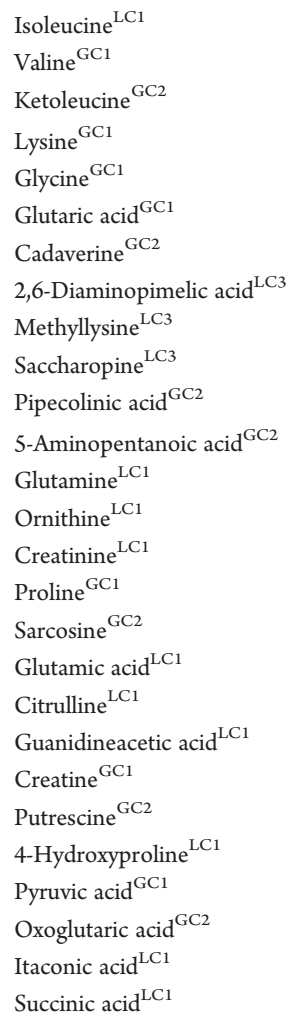


Table 1. Continued

\begin{tabular}{|c|c|c|}
\hline metabolic pathway & urinary metabolites & fecal metabolites \\
\hline Others & $\begin{array}{l}\text { Glycylprolylhydroxyproline } \\
\text { Citramalic acid }^{\mathrm{LC} 3} \\
\text { Aminomalonic acid }^{\mathrm{GC} 2} \\
\text { Argininic acid }^{\mathrm{LC} 3} \\
\text { Hydroxyglutaric acid }^{\mathrm{LC} 3} \\
\text { D-1-Piperideine-2-carboxylic acid } \\
\text { Dihydrodaidzein } \\
\text { Ur3 } \\
\text { Urobilin } \\
\text { N-Acetylcadaverine } \\
\text { N-Methylformamide } \\
\text { Ophthalmic acid } \\
\text { PC3 } \\
\text { Phenyllactic acid } \\
\text { Salicyluric acid } \\
\text { S-Succinyldihydrolipoamide } \\
\text { 4-Hydroxycyclohexylcarboxylic acid } \\
\text { Phenylalanylhydroxyproline } \\
\text { 3-(3-Hydroxyphenyl)propionic } \text { acid }^{\mathrm{LC} 3}\end{array}$ & $\begin{array}{l}\text { Glycylprolylhydroxyproline } \\
\text { Citramalic acid }^{\mathrm{LC} 1} \\
\text { Aminomalonic acid }^{\mathrm{LC} 3} \\
\text { Argininic acid }^{\mathrm{LC} 3} \\
\text { Hydroxyglutaric acid }^{\mathrm{GC} 2} \\
\text { D-1-Piperideine-2-carboxylic acid } \\
\text { Dihydrodaidzein } \\
\text { Uro3 } \\
\text { Urobilin } \\
\text { NC3 } \\
\text { N-Metylcadaverine } \\
\text { Ophthalformamide acid } \\
\text { PC3 } \\
\text { Phenyllactic acid } \\
\text { Salicyluric acid } \\
\text { S-Succinyldihydrolipoamide } \\
\text { 4-Hydroxycyclohexylcarboxylic acid } \\
\text { Phenylalanylhydroxyproline } \\
\text { 3-(3-Hydroxyphenyl) } \text { propionic acid }^{\text {LC2 }} \\
\text { Carnitine }\end{array}$ \\
\hline
\end{tabular}

${ }^{a}$ LC, identified from LC-MS data (exact mass); GC, identified from GC-MS data; 1, annotated with reference standards; 2 , annotated with National Institute of Standards and Technology (NIST) database; 3, annotated with Human Metabolome Database (HMDB).

metabolites that were most significantly altered in urine and feces in at least one day during the course of antibiotic treatment and recovery are listed in Table 1 with $p$ values of two-tailed unpaired $t$ test less than 0.05 . A complete list of differential metabolites containing 202 urinary metabolites and 223 fecal metabolites is provided in Supplementary Table 1 and 2 with information of $p$ value and fold change (FC) of mean intensity every day. Fifty representative metabolites were used to construct a heat-map, visualizing the time-dependent fluctuation of gut microbe-related metabolites (Figure 2). The heat-maps generated from a complete list of differential metabolites of urine and feces are provided in Supplementary Figure 2. The heat-maps visualized that the fluctuations in most of the metabolites in urine and feces samples were substantial at the first several days and attenuated in the recovery stage.

Altered Distribution of Metabolites Related to Gut Microbiota

Table 1 lists a number of aromatic substances, such as benzene and indole-containing substances, showing significant fluctuations (with large fold changes) both in urine and feces. The benzene-containing substances include $p$-hydroxyphenylacetic acid, $m$-hydrophenylacetic acid, hydroxycinnamic acid, phenylvaleric acid, and $p$-aminobenzoic acid. Hippuric acid in urine was of significantly lower level from day 1 to day 5 in the antibiotic group compared with the control group. Most of indole-containing substances including indoxyl sulfate, indoleacetic acid, indolecarboxylic acid, indoleacetaldehyde were decreased after antibiotic exposure. The two pie charts in Figure 3 illustrate the categories of the altered metabolites in urine and feces samples of antibiotic-perturbed rats. Tryptophan metabolism appeared to be significantly altered in the antibiotic group both in urine and feces. Tryptophan and tryptamine increased significantly in feces with antibiotic treatment as shown in Figure 4. Methyltryptamine and hydroxytryptophol were also increased while tryptophan decreased in urine. Many SCFAs were detected with lower levels both in urine and feces. SCFAs in feces were down-regulated to a much greater extent than those in urine. Several oligopeptides especially dipeptides and tripeptides significantly
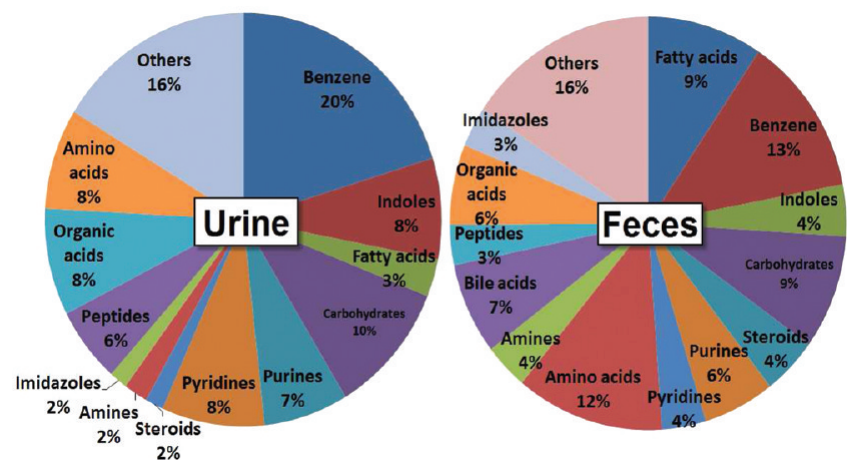

Figure 3. Classification of urinary and fecal metabolites derived from gut microbial-mammalian co-metabolism as pie charts shown.

increased in urine. Urobilin was significantly depleted both in urine and feces.

\section{DISCUSSION}

A number of different antibiotics have thus far been applied to the investigations of antibiotic perturbed gut microbiota, with a different effect of microbial perturbation. ${ }^{25-27}$ Imipenem is a $\beta$-lactam antibiotic with an extremely broad spectrum of activity against aerobic and anaerobic gram positive as well as gram negative bacteria, and therefore, was selected in our study to rapidly and completely remove the microbes from the GI tract. The antibiotic agent remains stable in the presence of $\beta$-lactamase (both penicillinase and cephalosporinase) and is a strong inhibitor of $\beta$-lactamases from gram-negative bacteria that are resistant to most $\beta$-lactam antibiotics. ${ }^{28}$ On the basis of its poor systemic bioavailability, Imipenem's direct impact is on the gut microbiota, instead of host metabolic pathways. A study on the analysis of urine samples collected from cynomolgus monkey dosed with imipenem revealed a significant increase of $\beta$-hydroxybutyrate and ketone bodies, indicating a disruption of energy metabolism resulting from the suppression of gut microflora. ${ }^{29}$ Imipenem, 

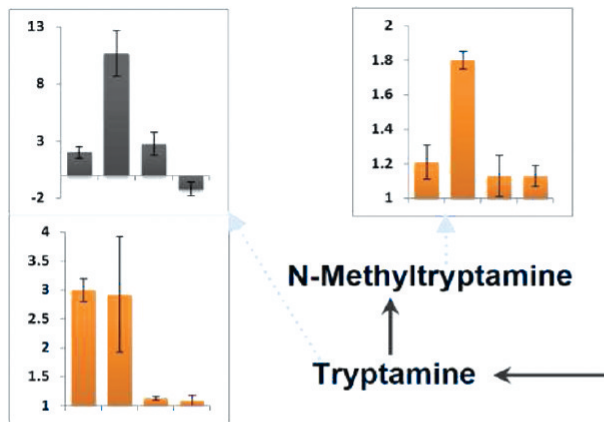

N-Methyltryptamine

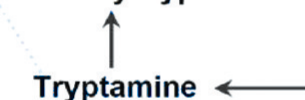

5-Hydroxyindoleacetic acid

Tryptamine

(a)

Indolepyruvic acid

Trophan

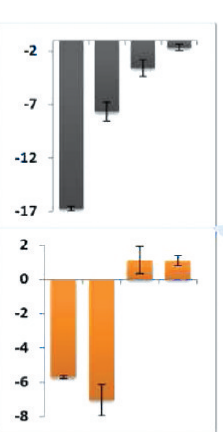

Indoleacetaldehyde $\longrightarrow$ Tryptophano

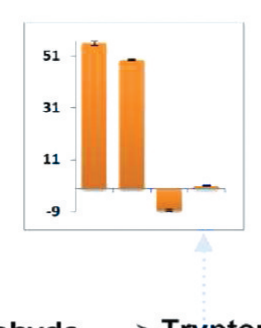

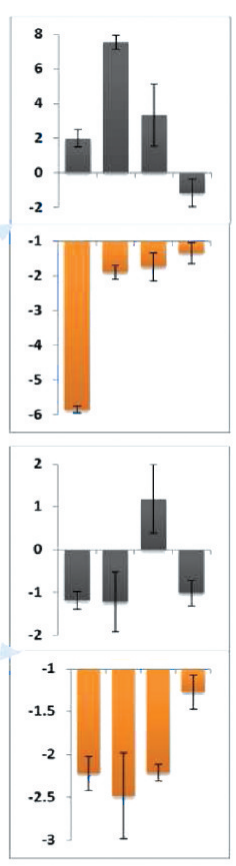
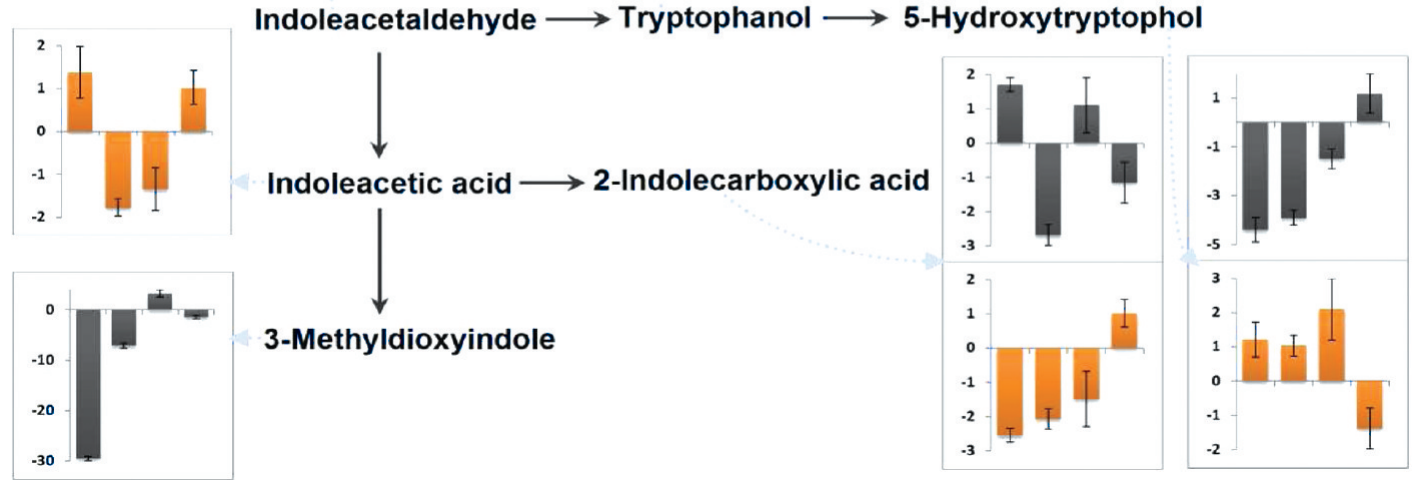

Figure 4. Dynamic alteration of tryptophan-indole metabolism. The bar plots show the fold change ( $y$ axis) of each metabolite in the pathway as the ratio of the concentration in the antibiotic group to that in the control group on days $1,3,5,8$ ( $x$ axis). The gray bars denote the fecal metabolites, while the orange bars denote the urinary metabolites.

cilastatin and their metabolites were rapidly excreted and, thus, not detected by mass spectrometry analysis from the samples collected at the time points of the experiment.

The global metabolic effects and dynamic changes of microbial composition in the antibiotic treated rats can be visualized from trajectories, PLS-DA scores plots and heat-maps in Figures 1 and 2. The trajectories of urinary and fecal metabolome displayed the dynamic changes in the antibiotic group during 19 days of experiment along the second principal component, and showed age-related physiological changes along the first pricinpal component in both groups. These systemic visualization plots suggest that experimental rats after a 4-day antibiotic treatment were able to regain the gut metabolic and ecological balance within a 2 -week recovery period. Since the fecal metabolic profile was a direct reflection of changes in microbial composition due to the antibiotic exposure, it appeared that the antibiotic intervention impacted fecal metabolites to a greater extent than urinary metabolites, from the number and statistical significance of variables (altered metabolites) detected. The total number of urinary and fecal metabolites involved in gut microbial-mammalian cometabolism discovered in our study was far greater than those reported so far. ${ }^{10,11,27}$ These altered metabolites are widely distributed across the mammalian metabolic system as shown in Supplementary Figure 3 (Supporting Information), many of which are mammalian metabolites involved in amino acids and glucose metabolism.

Tryptophan is believed to be associated with gut microbes as it is metabolized into indole by tryptophanase in intestinal bacteria, primarily, Escherichia coli, ${ }^{30}$ which is further absorbed into the blood from the intestine and metabolized to indoxyl sulfate in the liver, and then excreted into urine. With a transient elimination of most of the gut microbial population, important bacterial enzymes such as tryptophanase were rapidly decreased, leading to a strong perturbation of tryptophan and indole metabolic pathways. As a result, indoles were depleted in both urine and feces while tryptophan levels rose in feces (Figure 4). Tryptamine significantly increased both in urine and feces presumably due to the fact that less amount of tryptamine was transformed via microbial enzymes to indole-containing molecules while the production of tryptamine via the mammalian metabolic enzyme, trypthophan decarboxylase, remained unaffected. Another metabolite, 6-hydroxymelatonin sulfate, as the major metabolite of melatonin 
and a good marker of melatonin synthesis and secretion, ${ }^{31}$ increased in urine excretion. Recent studies have shown that indole modulates expression of proinflammatory genes, increases expression of anti-inflammatory genes, and strengthens epithelial cell barrier properties, suggesting that indole is a beneficial bacterial signal for intestinal epithelial cells. ${ }^{32}$ In the meantime, melatonin produced by the pineal gland in the brain and a variety of peripheral cells such as bone marrow, lymphocytes and epithelial cells ${ }^{33}$ was elevated as shown by 6-hydroxymelatonin elevation both in urine and feces. Since indole and melatonin are structurally similar to each other and may share certain biological functions such as antiproliferative properties, the abnormally high expression of melatonin observed in urine and feces may be directly associated with the depleted indole level after the gut microbial disruption for maintaining gut homeostasis. Additionally, several tryptophan and tyrosine metabolites, as neurotransmitters such as dopa, dopamine, norepinephrine, and epinephrine, were perturbed in urine and feces. These findings bring evidence that the microbial-mammalian co-metabolism is strongly associated with the gut-brain axis (GBA), a bidirectional neurohumoral communication system that integrates brain and GI functions. ${ }^{34}$ The GBA seems to regulate homeostasis of GI function, in that GI motility and epithelial functions determine the habitat of the microbiota, while changes induced by the central nervous system or the GI tract alter the habitat and perturb the intestinal microbiota. ${ }^{34}$

The metabolism of tyrosine and phenylalanine is believed to be associated with microbiota. ${ }^{27,35}$ Significant depletion of the postdose concentrations of hippuric acid (11-fold) and $p$-cresol ( 8 fold) from day 1 to day 5 in the antibiotic group and slight elevation at the end of the recovery period were observed in urine. Hippuric acid is a metabolite produced by intestinal microbes in the presense of quinic acid, ${ }^{27}$ formed by glycine conjugation of benzoate. The significant decrease of hippuric acid was accordance with the observation that Harrison et al. reported. ${ }^{29}$ Tyrosine and phenylalanine in feces were noted to be associated with urinary hippuric acid and phenylacetylglycine which are produced by the catabolism of these amino acids via microbiota. ${ }^{35}$ Hydroxyphenylacetic acid is produced from tyrosine involving microbial enzymes. That tyrosine altered only in one day in urine and feces and hydroxyphenylacetic acid altered almost every day even in the recovery period suggests that this bacterial-mediated synthesis was greatly impacted and their recovery was slow. As a result, the concentration of $p$-cresol, decarboxylated from $p$-hydroxyphenylacetic acid, was also altered. ${ }^{36}$

SCFAs are monocarboxylic acids with up to six carbon atoms, such as acetic acid, propionic acid, butyric acid, valeric acid, 2 -methylbutyric acid and isovaleric acid. This group of metabolites is probably an important "gateway" between intestinal bacteria and host metabolism in terms of mtebolic flux and volume, since SCFAs are primarily generated from the bacterial fermentation of carbohydrates in the colon, but also serve as intermediates and precursors of many metabolic activities, providing energy to muscle, kidney, heart and brain. ${ }^{37}$ With antibiotic perturbation, SCFA production by microbiota including bacteroidetes, firmicutes, actinobacteria, and proteobacteria is significantly suppressed. ${ }^{38}$ SCFAs especially butyric acid was found depleted in feces in the first several days of the experiment, and down-regulated in urine until late in the recovery period. Additionally, free fatty acids, caprylic acid, myristic acid, palmitic acid, and suberic acid were also found decreased in feces along with the depleted level of SCFAs.
A number of amino acids, the products of food breakdown and intermediates of catabolic metabolism with the involvement of gut microbes, fluctuated as a result of antibiotic exposure. For example, the histidine level rose and urocanate fell significantly in feces in the first day of antibiotic treatment as a direct result of microbial suppression, since histidine is metabolized to urocanic acid by means of an intestinal enzyme, histidase, produced by peptostreptococcs asaccharolyticus. ${ }^{39}$ Histidine and related degraded products in urine altered to a lesser extent than in feces in the first day. Urocanic acid altered in the same direction in urine but with a lower fold change compared to that in feces.

Several oligopeptides especially dipeptides and tripeptides were found elevated in urine of the antibiotic group, presumably due to the high expression of $\mathrm{H}^{+}$/peptide transporter PepT1, leading to the increased small peptides excretion through kidney as a result of transient gut microbial disruption. An important transport function expressed by gut epithelial cells is the absorption of small peptides from the diet by an apical membrane peptide transporter. ${ }^{40}$ Intestinal adaptation after inflammation is characterized by increased gut-mucosal growth, as reported that the $\mathrm{H}^{+}$/peptide transporter PepT1 expression was high in the colonic mucosa of patients with short-bowel syndrome (SBS), so that the large intestine has a mechanism for luminal di- and tripeptide transport, increasing the absorption of di- and tripeptides. ${ }^{41}$ Therefore, rapidly increased levels of dipeptides and tripeptides may be potential markers for intestinal dysbiosis.

Urobilin was also significantly down-regulated both in urine and feces. Urobilin, the degradation product of bilirubin, is one of the most important compounds of urobilinoids formed in the Gl tract by intestinal microbes. With antibiotic suppressed bilirubindegradation microbes, urobilinoids were lowered significantly as our results show. Urobilin was also down-regulated in urine, however not as significantly as in feces, due to the fact that urobilinoids underwent enterohepatic and enterosystemic circulation and only about $10-20 \%$ were reabsorbed and excreted in urine. ${ }^{42}$

The present methodological study aimed to investigate the feasibility of metabonomic profiling approach to capture a full spectrum of metabolic changes derived from gut microbial-host co-metabolism. The results of the study suggest that metagenomic compositional changes can be eventually monitored and probed using a metabonomic approach, providing that the two sets of changes can be correlated to each other.

\section{CONCLUSION}

Real time analysis of the small molecule metabolites derived from microbial-mammalian metabolic cooperation is essential for the understanding of the metabolic functions of the metagenome with tremendous implications for personalized healthcare strategies. The study identified a panel of urinary and fecal metabolites involved in gut microbial-mammalian co-metabolism using a combined $\mathrm{GC}-\mathrm{MS}$ and $\mathrm{LC}-\mathrm{MS}$ metabonomic profiling approach. This study also brings further evidence that the gut microbiota modulate the host systemic metabolism involving tryptophan, lipids, amino acids, and possibly a compensatory mechanism of indole-melatonin production. It appeared that the recovery of the global metabonomic changes takes about two-weeks. With the use of complementary LC-MS and GC-MS platforms, $\sim 800$ endogenous metabolite standards and other web-based metabolite databases in this study, we identified a panel of 202 urinary and 223 fecal metabolites $(p<0.05$ in at 
least one day) significantly altered as a readout of a gut microbial-mammalian co-metabolism, many of which have not been previously reported.

\section{ASSOCIATED CONTENT}

\section{S Supporting Information}

Procedure of urine and feces samples preparation; LC-MS and GC-MS profiling methods; trajectories of different data sets from urine and feces analyzed by LC-MS and GC-MS; heatmaps of all differential metabolites derived in urine and feces; Overview of related metabolites in the whole mammalian metabolome; all of the urinary and fecal metabolites associated with gut microbial-mammalian co-metabolism. This material is available free of charge via the Internet at http://pubs.acs.org.

\section{AUTHOR INFORMATION}

\section{Corresponding Author}

*Wei Jia, Department of Nutrition, University of North Carolina at Greensboro, North Carolina Research Campus, Kannapolis, North Carolina 28081. Phone: 1-704-250-5803. Fax: 1-704-2505809. E-mail: w_jia@uncg.edu. Weiping Jia, Department of Endocrinology and Metabolism, Shanghai Jiao Tong University Affiliated Sixth People's Hospital, 600 Yishan Road, Shanghai 200233, China. Tel: +86-21-6436-9181. Fax: +86-21-6436-8031. E-mail: wpjia@sjtu.edu.cn.

\section{ACKNOWLEDGMENT}

This work was funded by the National Basic Research Program of China (2007CB914700).

\section{ABBREVIATIONS}

FC, fold change; GBA, gut-brain axis; GC-MS, gas chromatography-mass spectrometry; GI, gastrointestinal; LC-MS, liquid chromatography-mass spectrometry; MS, mass spectrometry; NMR, nuclear magnetic resonance; PLS-DA, partial least quared-discriminant analysis; Q-TOF, quadrupole-timeof-flight; Rt, retention time; SBS, short-bowel syndrome; SCFAs, short-chain fatty acids; SPF, specific pathogen-free; UPLC, ultra performance liquid chromatography

\section{REFERENCES}

(1) Knight, D. J. W.; Girling, K. J. Gut flora in health and disease. Lancet 2003, 361 (9371), 1831-1831.

(2) Hooper, L. V.; Gordon, J. I. Commensal host-bacterial relationships in the gut. Science 2001, 292 (5519), 1115-1118.

(3) Nicholson, J. K.; Holmes, E.; Wilson, I. D. Gut microorganisms, mammalian metabolism and personalized health care. Nat. Rev. Microbiol. 2005, 3 (5), 431-438.

(4) Jia, W.; Li, H. K.; Zhao, L. P.; Nicholson, J. K. Gut microbiota: a potential new territory for drug targeting. Nat. Rev. Drug Discovery 2008, 7 (2), 123-129.

(5) Zaneveld, J.; Turnbaugh, P. J.; Lozupone, C.; Ley, R. E.; Hamady, M.; Gordon, J. I.; Knight, R. Host-bacterial coevolution and the search for new drug targets. Curr. Opin. Chem. Biol. 2008, 12 (1), 109-114.

(6) Gill, S. R.; Pop, M.; DeBoy, R. T.; Eckburg, P. B.; Turnbaugh, P. J.; Samuel, B. S.; Gordon, J. I.; Relman, D. A.; Fraser-Liggett, C. M.; Nelson, K. E. Metagenomic analysis of the human distal gut microbiome. Science 2006, 312 (5778), 1355-1359.
(7) Hugenholtz, P.; Goebel, B. M.; Pace, N. R. Impact of cultureindependent studies on the emerging phylogenetic view of bacterial diversity. J. Bacteriol. 1998, 180 (18), 4765-4774.

(8) Shen, J.; Zhang, B. R.; Wei, G. F.; Pang, X.; Wei, H.; Li, M.; Zhang, Y.; Jia, W.; Zhao, L. P. Molecular profiling of the Clostridium leptum subgroup in human fecal microflora by PCR-denaturing gradient gel electrophoresis and clone library analysis. Appl. Environ. Microbiol. 2006, 72 (8), 5232-5238.

(9) Pang, X. Y.; Hua, X. G.; Yang, Q.; Ding, D. H.; Che, C. Y.; Cui, L.; Jia, W.; Bucheli, P.; Zhao, L. P. Inter-species transplantation of gut microbiota from human to pigs. Isme J. 2007, 1 (2), 156-162.

(10) Li, M.; Wang, B. H.; Zhang, M. H.; Rantalainen, M.; Wang, S. Y.; Zhou, H. K.; Zhang, Y.; Shen, J.; Pang, X. Y.; Zhang, M. L.; Wei, H.; Chen, Y.; Lu, H. F.; Zuo, J.; Su, M. M.; Qiu, Y. P.; Jia, W.; Xiao, C. N.; Smith, L. M.; Yang, S. L.; Holmes, E.; Tang, H. R.; Zhao, G. P.; Nicholson, J. K.; Li, L. J.; Zhao, L. P. Symbiotic gut microbes modulate human metabolic phenotypes. Proc. Natl. Acad. Sci. U.S.A. 2008, 105 (6), $2117-2122$.

(11) Nicholls, A. W.; Mortishire-Smith, R. J.; Nicholson, J. K. NMR spectroscopic-based metabonomic studies of urinary metabolite variation in acclimatizing germ-free rats. Chem. Res. Toxicol. 2003, 16 (11), 1395-1404.

(12) Wang, X. Y.; Su, M. M.; Qiu, Y. P.; Ni, Y.; Zhao, T.; Zhou, M. M.; Zhao, A. H.; Yang, S. L.; Zhao, L. P.; Jia, W. Metabolic regulatory network alterations in response to acute cold stress and ginsenoside intervention. J. Proteome Res. 2007, 6 (9), 3449-3455.

(13) Bao, Y. Q.; Zhao, T.; Wang, X. Y.; Qiu, Y. P.; Su, M. M.; Jia, W. P.; Jia, W. Metabonomic Variations in the Drug-Treated Type 2 Diabetes Mellitus Patients and Healthy Volunteers. J. Proteome Res. 2009, 8 (4), 1623-1630.

(14) Fiehn, O. Metabolomics - the link between genotypes and phenotypes. Plant Mol.Biol. 2002, 48 (1-2), 155-171.

(15) Nicholson, J. K.; Lindon, J. C.; Holmes, E. 'Metabonomics': understanding the metabolic responses of living systems to pathophysiological stimuli via multivariate statistical analysis of biological NMR spectroscopic data. Xenobiotica 1999, 29 (11), 1181-1189.

(16) Claus, S. P.; Tsang, T. M.; Wang, Y.; Cloarec, O.; Skordi, E.; Martin, F.-P.; Rezzi, S.; Ross, A.; Kochhar, S.; Holmes, E.; Nicholson, J. K. Systemic multicompartmental effects of the gut microbiome on mouse metabolic phenotypes. Mol. Syst. Biol. 2008, 4, 219.

(17) Martin, F. P. J; Dumas, M. E.; Wang, Y. L.; Legido-Quigley, C.; Yap, I. K. S.; Tang, H. R.; Zirah, S.; Murphy, G. M.; Cloarec, O.; Lindon, J. C.; Sprenger, N.; Fay, L. B.; Kochhar, S.; van Bladeren, P.; Holmes, E.; Nicholson, J. K. A top-down systems biology view of microbiomemammalian metabolic interactions in a mouse model. Mol. Syst. Biol. 2007, 3, 112 .

(18) Martin, F. P. J; Wang, Y.; Yap, I. K. S.; Sprenger, N.; Lindon, J. C.; Rezzi, S.; Kochhar, S.; Holmes, E.; Nicholson, J. K. Topographical variation in murine intestinal metabolic profiles in relation to microbiome speciation and functional ecological activity. J. Proteome Res. 2009, 8 (7), 3464-3474.

(19) Qiu, Y. P.; Cai, G. X.; Su, M. M.; Chen, T. L.; Zheng, X. J.; Xu, Y.; Ni, Y.; Zhao, A. H.; Xu, L. X.; Cai, S. J.; Jia, W. Serum metabolite profiling of human colorectal cancer using GC-TOFMS and UPLCQTOFMS. J. Proteome Res. 2009, 8 (10), 4844-4850.

(20) Xie, G. X.; Zheng, X. J.; Qi, X.; Cao, Y.; Chi, Y.; Su, M. M.; Ni, Y.; Qiu, Y. P.; Liu, Y. M.; Li, H. K.; Zhao, A. H.; Jia, W. Metabonomic evaluation of melamine-induced acute renal toxicity in rats. J. Proteome Res. 2009, 9 (1), 125-133.

(21) Gao, X. F.; Pujos-Guillot, E.; Sebedio, J. L. Development of a quantitative metabolomic approach to study clinical human fecal water metabolome based on trimethylsilylation derivatization and GC/MS analysis. Anal. Chem. 2010, 82 (15), 6447-6456.

(22) Jonsson, P.; Johansson, A. I.; Gullberg, J.; Trygg, J.; A, J.; Grung, B.; Marklund, S.; Sjostrom, M.; Antti, H.; Moritz, T. High-throughput data analysis for detecting and identifying differences between samples in GC/MS-based metabolomic analyses. Anal. Chem. 2005, 77 (17), 5635-5642. 
(23) Shannon, P.; Markiel, A.; Ozier, O.; Baliga, N. S.; Wang, J. T.; Ramage, D.; Amin, N.; Schwikowski, B.; Ideker, T. Cytoscape: A software environment for integrated models of biomolecular interaction networks. Genome Res. 2003, 13 (11), 2498-2504.

(24) Holmes, E.; Bonner, F. W.; Sweatman, B. C.; Lindon, J. C.; Beddell, C. R.; Rahr, E.; Nicholson, J. K. Nuclear-magnetic-resonance spectroscopy and pattern-recognition analysis of the biochemical processes associated with the progression of and recovery from nephrotoxic lesions in the rat induced by mercury(II) chloride and 2-bromoethanamine. Mol. Pharmacol. 1992, 42 (5), 922-930.

(25) Dethlefsen, L.; Relman, D. A. Incomplete recovery and individualized responses of the human distal gut microbiota to repeated antibiotic perturbation. Proc. Natl. Acad. Sci. U.S.A. 2011, 108, 4554-4561.

(26) Willing, B. P.; Russell, S. L.; Finlay, B. B. Shifting the balance: antibiotic effects on host-microbiota mutualism. Nat. Rev. Microbiol. 2011, 9 (4), 233-243.

(27) Yap, I. K. S.; Li, J. V.; Saric, J.; Martin, F. P.; Davies, H.; Wang, Y. L.; Wilson, I. D.; Nicholson, J. K.; Utzinger, J.; Marchesi, J. R.; Holmes, E. Metabonomic and microbiological analysis of the dynamic effect of vancomycin-induced gut microbiota modification in the mouse. J. Proteome Res. 2008, 7 (9), 3718-3728.

(28) Clissold, S. P.; Todd, P. A.; Campoli-Richards, D. M. Imipenem/cilastatin. A review of its antibacterial activity, pharmacokinetic properties and therapeutic efficacy. Drugs 1987, 33 (3), 183-241.

(29) Harrison, M. P.; Jones, D. V.; Pickford, R. J.; Wilson, I. D. betaHydroxybutyrate: a urinary marker of imipenem induced nephrotoxicity in the cynomolgus monkey detected by high field 1H NMR spectroscopy. Biochem. Pharmacol. 1991, 41 (12), 2045-9.

(30) Botsford, J. L.; Demoss, R. D. Escherichia-coli tryptophanase in enteric environment. J. Bacteriol. 1972, 109 (1), 74.

(31) Arendt, J.; Bojkowski, C.; Franey, C.; Wright, J.; Marks, V. Immunoassay of 6-hydroxymelatonin sulfate in human - plasma and urine - abolition of the urinary 24-h rhythm with atenolo. J. Clin. Endocrinol. Metab. 1985, 60 (6), 1166-1173.

(32) Bansal, T.; Alaniz, R. C.; Wood, T. K.; Jayaraman, A. The bacterial signal indole increases epithelial-cell tight-junction resistance and attenuates indicators of inflammation. Proc. Natl. Acad. Sci. U.S.A. 2010, 107 (1), 228-233.

(33) Cardinali, D. P. Melatonin - a mammalian pineal hormone. Endocr. Rev. 1981, 2 (3), 327-346.

(34) Collins, S. M.; Bercik, P. The relationship between intestinal microbiota and the central nervous system in normal gastrointestinal function and disease. Gastroenterology 2009, 136 (6), 2003-2014.

(35) Diaz, E.; Ferrandez, A.; Prieto, M. A.; Garcia, J. L. Biodegradation of aromatic compounds by Escherichia coli. Microbiol. Mol. Biol. Rev. 2001, 65 (4), 523.

(36) Smith, E. A.; Macfarlane, G. T. Formation of phenolic and indolic compounds by anaerobic bacteria in the human large intestine. Microb. Ecol. 1997, 33 (3), 180-188.

(37) Cook, S. I.; Sellin, J. H. Review article: short chain fatty acids in health and disease. Aliment.y Pharmacol. Ther. 1998, 12 (6), 499-507.

(38) Hammer, H. F.; Fine, K. D.; Santaana, C. A.; Porter, J. L.; Schiller, L. R; Fordtran, J. S. Carbohydrate malasorption - its measurement and its contribution of diarrhea. J. Clin. Invest. 1990, 86 (6), 1936-1944.

(39) Whiteley, H. R. Fermentation of amino acids by micrococcusaerogenes. J. Bacteriol. 1957, 74 (3), 324-330.

(40) Gilbert, E. R.; Wong, E. A.; Webb, K. E. Board-invited review: Peptide absorption and utilization: Implications for animal nutrition and health. J. Anim. Sci. 2008, 86 (9), 2135-2155.

(41) Ziegler, T. R.; Fernandez-Estivariz, C.; Gu, L. H.; Bazargan, N.; Umeakunne, K.; Wallace, T. M.; Diaz, E. E.; Rosado, K. E.; Pascal, R. R.; Galloway, J. R.; Wilcox, J. N.; Leader, L. M. Distribution of the H+/ peptide transporter PepT1 in human intestine: up-regulated expression in the colonic mucosa of patients with short-bowel syndrome. Am. J. Clin. Nutr. 2002, 75 (5), 922-930.

(42) Schmidt, N. A.; Scholtis, R. J. Urobilin in urine. Clin. Chim. Acta 1964, $10(6), 574$. 